\title{
Positively Curved Combinatorial 3-Manifolds
}

\author{
Aaron Trout \\ Department of Mathematics \\ Chatham University, Pittsburgh PA, USA \\ atrout@chatham.edu
}

Submitted: Dec 5, 2006; Accepted: Mar 18, 2010; Published: Mar 29, 2010

Mathematics Subject Classifications: 52C99, 53A99, 57M99

\begin{abstract}
We present two theorems in the "discrete differential geometry" of positively curved spaces. The first is a combinatorial analog of the Bonnet-Myers theorem:

- A combinatorial 3-manifold whose edges have degree at most five has edgediameter at most five.

When all edges have unit length, this degree bound is equivalent to an angle-deficit along each edge. It is for this reason we call such spaces positively curved.

Our second main result is analogous to the sphere theorems of Toponogov [12] and Cheng [2]:

- A positively curved 3-manifold, as above, in which vertices $v$ and $w$ have edgedistance five is a sphere whose triangulation is completely determined by the structure of $L k(v)$ or $L k(w)$.

In fact, we provide a procedure for constructing a maximum diameter sphere from a suitable $L k(v)$ or $L k(w)$.

The compactness of these spaces (without an explicit diameter bound) was first proved via analytic arguments in a 1973 paper by David Stone. Our proof is completely combinatorial, provides sharp bounds, and follows closely the proof strategy for the classical results.
\end{abstract}

\section{Introduction}

The relationship between the curvature of a Riemannian (or semi-Riemannian) space and its topology is of central interest to differential geometers, topologists, and physicists. The classical results in this area are numerous, beautiful, and have inspired an enormous amount of subsequent research. One currently active branch of this venerable tree seeks combinatorial analogs to these classical theorems and concepts. Recent work along these 
lines can be found in [1], [3], [4], [6], [7], [10] and [11]. Here we present combinatorial versions of the Bonnet-Myers theorem, and the associated maximum-diameter sphere theorems of Toponogov [12] and Cheng [2].

\section{Overview of Results and Preliminary Definitions}

This paper will investigate the geometry of combinatorial manifolds. Briefly stated, a (boundaryless) combinatorial $n$-manifold is a simplicial complex in which the link of each $k$-simplex is an $(n-k-1)$-sphere. The category of such spaces is equivalent to the category of piecewise-linear (PL) manifolds and, for $n \leqslant 4$, to the smooth and topological categories. We emphasize, however, that our results depend only on the structure of the manifold as an abstract simplicial complex and not on any additional PL or smooth structure.

Our first main theorem is a combinatorial version of the classical Bonnet-Myers theorem:

Theorem 1.1 Suppose $M^{n}$ is a connected, boundaryless, combinatorial n-manifold in which each $(n-2)$-simplex has degree at most $\epsilon(n)$ where

$$
\epsilon(n)= \begin{cases}5 & n=2,3 \\ 4 & n \geqslant 4\end{cases}
$$

Then $M^{n}$ is compact and has edge-diameter at most $\delta(n)$ where

$$
\delta(n)= \begin{cases}3 & n=2 \\ 5 & n=3 \\ 2 & n \geqslant 4 .\end{cases}
$$

The degree of a simplex $\sigma \in M^{n}$, denoted $\operatorname{deg}(\sigma)$, is the number of $n$-simplices in $M^{n}$ having $\sigma$ as a face. The edge-diameter of $M^{n}$, written $\operatorname{diam}_{1}\left(M^{n}\right)$, is the minimum number of edges needed to connect any vertex in $M^{n}$ to any other. A combinatorial manifold which satisfies the degree bounds in Theorem 1.1 will be called positively curved.

Why do we refer to such spaces as positively curved? If we endow $M^{n}$ with the PLmetric with unit length edges, the dihedral angles in each $n$-simplex are all $\cos ^{-1}\left(\frac{1}{n}\right)$. Therefore, the total angle around each $(n-2)$-simplex $\sigma$ is $\operatorname{deg}(\sigma) \cdot \cos ^{-1}\left(\frac{1}{n}\right)$. The degree bound $\epsilon(n)$ is the largest which guarantees this total angle is less than $2 \pi$. In the Riemannian setting such an angle deficit is intimately related to positive curvature.

Since $\mathbb{R}^{2}$ and $\mathbb{R}^{3}$ admit triangulations where the codimension- 2 simplices have degree at most six, the hypotheses cannot be weakened for $n \leqslant 3$. In fact, in [1] it is shown that any closed orientable 3-manifold admits a triangulation with edges of degree 4,5 or 6 . We suspect, but have no proof, that weakening the hypothesis for $n \geqslant 4$ would also lead to non-compact manifolds.

Our second main result is analogous to the rigid sphere theorems of Toponogov [12] and Cheng [2]: 
Theorem 1.2 Let $M$ be a positively curved combinatorial n-manifold.

1. If vertices $v, w \in M$ have edge-distance $\delta(n)$ then $M$ is a sphere.

2. If $M^{\prime}$ is another such manifold with vertices $v^{\prime}, w^{\prime}$ at edge-distance $\delta(n)$ and there exists a simplicial isomorphism $\Psi: L k(v) \cong L k\left(v^{\prime}\right)$ then $\Psi$ extends to a simplicial isomorphism $M \cong M^{\prime}$.

3. For each $(n-1)$-sphere $L$ with $(n-3)$-simplices of degree at most $\epsilon(n)$, we explicitly construct a positively curved $M$ with vertices $v$ and $w$ at edge-distance $\delta(n)$ and $L k(v)=L$.

The edge-distance between vertices $v, w \in M^{n}$ is the minimum number of edges needed to connect them and will be denoted by $d_{1}(v, w)$.

This paper will prove the $n=3$ case of the two main theorems. For $n=2$ the results are classically known and the $n \geqslant 4$ cases follow from the classification in [13]. The compactness of positively curved combinatorial 3-manifolds (without an explicit diameter bound) was first proved via analytic methods in a 1973 paper, [11], by David Stone. Our proof is completely combinatorial, provides sharp bounds, and follows closely the proof strategy for the classical results.

\section{Hops and Jumps}

Though our final results involve paths containing only edges, the proof will use a slightly expanded set of paths. All these will be straight lines when restricted to an individual simplex. In what follows, we use $\hat{\sigma}$ to denote the barycenter of a simplex $\sigma$.

Definition 2.1 (Hops) Consider an $(n-1)$-simplex $\tau \in M^{n}$ and the two $n$-simplices $v_{1} * \tau$ and $\tau * v_{2}$ where $v_{1}$ and $v_{2}$ are vertices. The PL-path from $v_{1}$ through $\hat{\tau}$ to $v_{2}$ will be called an n-dimensional hop from $v_{1}$ to $v_{2}$ (or an $n$-hop, or just a hop). We will say that $\tau$ and the hop are transverse to each other. See Figure 1.

A nice consequence of this definition is the following fact, given without proof.

Lemma 2.2 Suppose $v \in M^{n}$ is a vertex. Vertices $w_{1}, w_{2} \in L k(v)$ are connected by an $n$-hop within $\overline{S t(v)}$ if and only if they are connected by an $(n-1)$-hop within Lk $(v)$.

In dimension three it will be convenient to add another type of PL-path. See Figure 2 for an illustration.

Definition 2.3 (Jump) Consider a 3-simplex $e_{1} * e_{2}$ and 2-simplices $v_{1} * e_{1}$ and $e_{2} * v_{2}$, where the $e_{i}$ are edges and the $v_{i}$ vertices. The PL-path from $v_{1}$ through $\hat{e_{1}}$ and $\hat{e_{2}}$ to $v_{2}$ will be called a jump from $v_{1}$ to $v_{2}$. We will say that the jump and each edge $e_{i}$ are transverse to each other. See Figure 2. 



Figure 1: Two and three dimensional hops

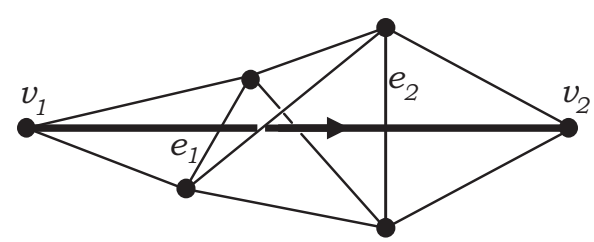

Figure 2: A jump

The length of a hop or jump will be its length as a PL-path, computed using the PLmetric in which all edges have unit length. Using some Euclidean geometry these values can be easily calculated.

Fact 2.4 An n-dimensional hop has length $H_{n}=\sqrt{2+\frac{2}{n}}$ and a jump has length $J=$ $\sqrt{3}+\frac{1}{2} \sqrt{2}$.

Restricting ourselves to paths containing only edges, hops, and (in dimension three) jumps gives a distance function on the vertices of $M^{n}$ which we denote $d$. The distance between sets of simplices $A, B \subset M^{n}$ will be given by:

$$
d(A, B)=\min \{d(v, w) \mid v \in \bar{A}, w \in \bar{B} \text { are vertices }\} .
$$

Diameters and other functions derived from $d$ will have their familiar notations.

We will use the following terminology to refer to paths which minimize or almost minimize distance.

Definition 2.5 If the length of a path $P$ equals the distance between its endpoints then we call it minimal. If each proper subpath of $P$ is minimal we say that $P$ is almost minimal.

Note that a minimal path is necessarily almost-minimal, but the converse need not hold. Also note that while a path containing a single edge must be minimal, a path containing a single hop (or jump) may not be.

Consider a path containing a single edge, hop or jump. For an edge, the first two simplices the path passes through uniquely determine the remainder of the path. For hops and jumps this is no longer the case. However, minimal hops and jumps continue to have this useful property. 
Lemma 2.6 Suppose $P$ and $Q$ are minimal paths each containing a single edge, hop or jump. If $P$ and $Q$ pass through the same initial two simplices then the paths are identical.

PRoOF. Clearly $P$ and $Q$ are either both edges, both hops or both jumps. Edges are by definition uniquely determined by their initial two simplices. So, suppose $P$ and $Q$ are hops both of which begin on the vertex $v_{1}$ and then passing into the $n$-simplex $v_{1} * \tau$ where $\tau$ is an $(n-1)$-simplex. Since $M$ is a boundaryless combinatorial $n$-manifold, the star of $\tau$ contains exactly two $n$-simplices, $v_{1} * \tau$ and $v_{2} * \tau$. If $P$ and $Q$ are minimal they must end on $v_{2}$ and are therefore identical as desired. This completes the proof for $n \neq 3$.

When $n=3$ we must also consider jumps. Let $P$ and $Q$ be minimal jumps both of which begin on the vertex $v_{1}$ and then pass into the 2 -simplex $v_{1} * e_{1}$, where $e_{1}$ is the first edge transverse to each jump. The remainder of each jump is determined by selecting the other transverse edge $e_{2} \in \operatorname{Lk}\left(e_{1}\right)$ and the final vertex $v_{2} \in \operatorname{Lk}\left(e_{2}\right)$. If $\operatorname{deg}\left(e_{1}\right) \leqslant 4$ then $d\left(v_{1}, e_{2}\right) \leqslant 1$ and by the structure of a jump we would have $d\left(v_{1}, v_{2}\right) \leqslant 1+1<J$, contradicting minimality of the jump. Therefore, $\operatorname{deg}\left(e_{1}\right)=5$ and there is exactly one choice of $e_{2}$. A similar argument shows that $\operatorname{deg}\left(e_{2}\right)=5$ and there is exactly one choice of ending vertex $v_{2}$. Therefore, $P$ and $Q$ are identical as desired.

We will need notation for the vertices along a path and also the order in which the hops, jumps and edges occur.

Definition 2.7 Let $P^{v}$ be the ordered list of vertices which $P$ visits. Vertices other than the first and last we call internal. $P^{l}$ will denote the ordered list containing a "1", " $H_{n}$ ", or "J" according to the order in which the edges, hops and jumps occur.

Note that $P^{v}$ does not necessarily uniquely determine the path $P$ or even the list $P^{l}$.

\section{Two Dimensional Case}

Suppose $M^{2}$ is a positively curved combinatorial surface. The complete census of such surfaces is classically known. Therefore, the $n=2$ case of our main theorems can be proved by inspection. We will also need some additional results concerning these surfaces, which can also be proved by inspection.

The first result we need concerns the structure of minimal paths and the structure of the surface along such paths.

Lemma 3.1 If $P$ is a minimal path with one internal vertex $x$ then $\operatorname{deg}(x)=5$ and $P$ has length $1+H_{2}$. Moreover, given the initial hop or edge in $P$ the remainder is uniquely determined.

Notice that according to Lemma 3.1, if a non-trivial minimal path in $M^{2}$ can be extended to a longer minimal path then this extension is unique, just as in the Riemannian setting.

It turns out that a minimal path in a positively curved surface can have at most one internal vertex. This means we have: 

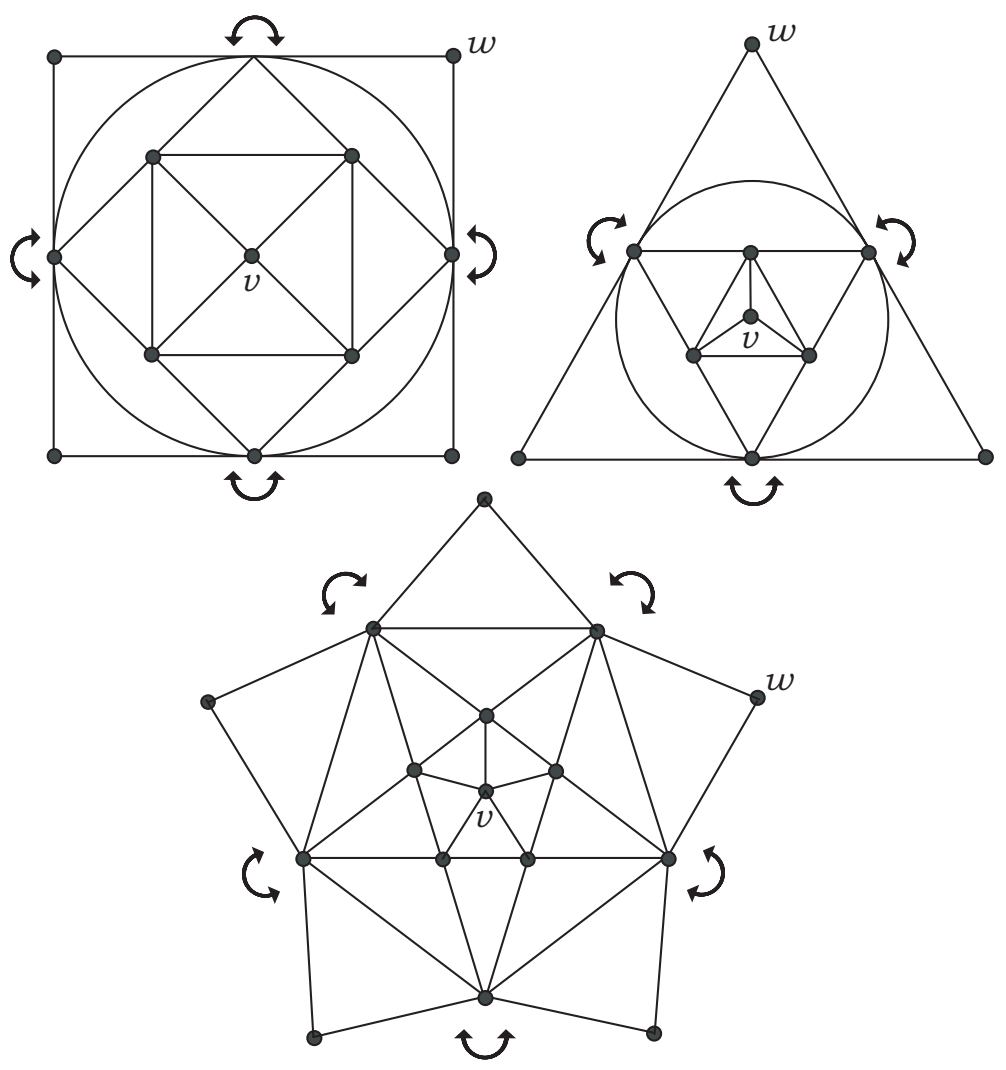

Figure 3: All the positively curved surfaces with $d_{1}(v, w)=3$. The arrows indicate that the corresponding edges are glued together.

Corollary $3.2 d(v, w) \in\left\{0,1, H_{2}, 1+H_{2}\right\}$ for any vertices $v, w \in M^{2}$. Moreover, $d(v, w)=1+H_{2}$ if and only if $d_{1}(v, w)=3$.

Also, the vertex at maximum distance is unique:

Corollary 3.3 For a fixed vertex $v$, we have $d_{1}(v, w)=3$ for at most one vertex $w$.

The positively curved surfaces of maximum diameter are depicted in Figure 3. For these surfaces we have the following facts:

Corollary 3.4 Suppose $d_{1}(v, w)=3$ for vertices $v, w$ in a positively curved surface $M^{2}$. Then, we have:

1. $\operatorname{deg}(v)=\operatorname{deg}(w)$.

2. Any minimal hop beginning on $v$ ends on a vertex in $L k(w)$.

3. Any vertex in $L k(v)$ is the beginning of a minimal hop to $w$. 


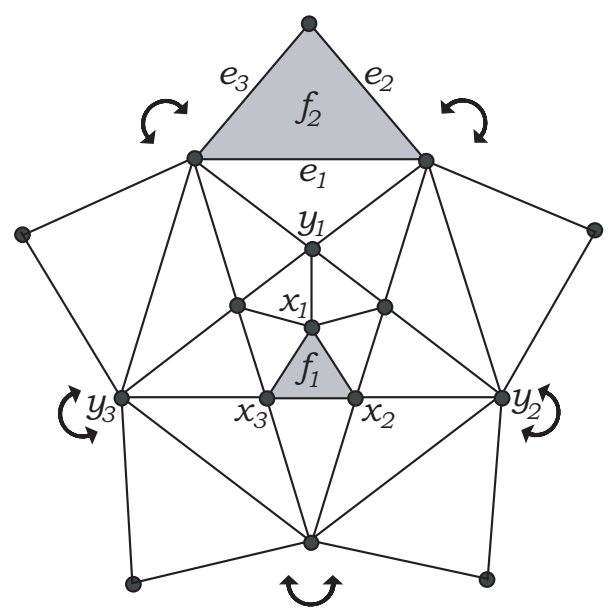

Figure 4: The $f_{i}, x_{i}, y_{i}, e_{i}$ from Lemma $3.6(1)$ and the $f_{i}$ from Lemma 3.5 (1)

The following two diameter properties uniquely characterize the icosahedron among the positively curved surfaces.

Lemma 3.5 For all 2-simplices $f_{1}, f_{2}$ and 1-simplices $e_{1}, e_{2}$ in a positively curved surface $M^{2}$ we have

1. $d\left(f_{1}, f_{2}\right) \leqslant H_{2}$, and

2. $d\left(S t\left(e_{1}\right), e_{2}\right) \leqslant H_{2}$.

Moreover, for fixed $f_{1}$ (or $e_{1}$ ) at most one $f_{2}$ (or $e_{2}$ ) gives equality, and this occurs only when $M^{2}$ is an icosahedron. See Figures 4 and 5.

When equality occurs in Lemma 3.5 there are some specific simplices to which we will need to refer.

Lemma 3.6 Let $M^{2}$ be an icosahedron.

1. Suppose $d\left(f_{1}, f_{2}\right)=H_{2}$ for 2-simplices $f_{1}, f_{2} \in M^{2}$. For each vertex $x_{i} \prec f_{1}$ there is a unique edge $e_{i} \prec f_{2}$ and vertex $y_{i} \in \operatorname{Lk}\left(e_{i}\right)$ so that $\left[x_{i}, y_{i}\right]$ is an edge. Similarly, each $e_{i} \prec f_{2}$ gives unique $y_{i} \in \operatorname{Lk}\left(e_{i}\right)$ and $x_{i} \prec f_{1}$ such that $\left[x_{i}, y_{i}\right]$ is an edge. See Figure 4 .

2. Suppose d $\left(S t\left(e_{1}\right), e_{2}\right)=H_{2}$ for edges $e_{1}, e_{2} \in M^{2}$. An edge connects each vertex in $L k\left(e_{1}\right)$ to exactly one vertex in $L k\left(e_{2}\right)$ (and vice-versa). See Figure 5.

Finally, we mention a convenient fact which lets us apply lower dimensional results to the higher dimensional cases.

Lemma 3.7 If $\operatorname{deg}(\sigma * \tau)=k$ within $M^{n}$ then $\operatorname{deg}(\tau)=k$ within $L k(\sigma)$. So, if $M^{n}$ is positively curved then so is each $L k(\sigma) \subset M^{n}$. 


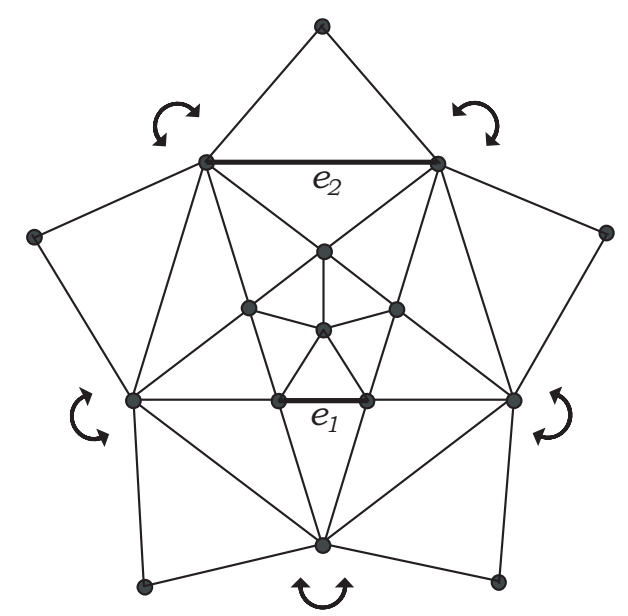

Figure 5: The $e_{i}$ from Lemma $3.5(2)$ and Lemma $3.6(2)$

\section{Combinatorial Bonnet-Myers Theorem}

In this section we prove the $n=3$ case of our first main theorem, which we restate here for the readers convenience.

Theorem 1.1 A combinatorial 3-manifold with edges of degree at most five has edge diameter at most five.

So, let $M^{3}$ be such a manifold. Our main argument begins by elucidating the structure of $M^{3}$ near an internal vertex of a minimal path.

Lemma 4.1 Suppose $P$ is a minimal path with $P^{v}=\left(v_{0}, v_{1}, v_{2}\right)$. Within the positively curved surface $L=L k\left(v_{1}\right)$ we know:

1. If $P$ is a two edge path then $d^{L}\left(v_{0}, v_{2}\right)=1+H_{2}$.

2. If $P$ is a two hop path then $d^{L}\left(f_{1}, f_{2}\right)=H_{2}$ where the 2-simplices $f_{1}, f_{2} \in L k\left(v_{1}\right)$ are transverse to the hops.

3. If $P$ is a two jump path then $d^{L}\left(S t^{L}\left(e_{1}\right), e_{2}\right)=H_{2}$ where the edges $e_{1}, e_{2} \in L k\left(v_{1}\right)$ are transverse to the jumps.

In each case, given $v_{0}, f_{1}$, or $e_{1}$ the corresponding $v_{2}, f_{2}$, or $e_{2}$ is uniquely determined. In cases (2) and (3), $L k\left(v_{1}\right)$ is an icosahedron.

NOTATION: We write $d^{L}$ to denote the distance within the 2 -sphere $L$ rather than in $M^{3}$. Similarly, $S t^{L}(\sigma) \equiv S t(\sigma) \cap L$ and $L k^{L}(\sigma) \equiv L k(\sigma) \cap L$ are the star and link of $\sigma$ respectively, within $L$.

PROOF. $\quad L=L k\left(v_{1}\right)$ is a positively curved surface by Lemma 3.7.

PART (1): If $d^{L}\left(v_{0}, v_{2}\right)$ were smaller, Corollary 3.2 and Lemma 2.2 would imply that $d\left(v_{0}, v_{2}\right) \leqslant H_{3}$, contradicting the minimality of $P$. Thus, $d^{L}\left(v_{0}, v_{2}\right)=1+H_{2}$ as desired. 
PART (2): We cannot have $d^{L}\left(f_{1}, f_{2}\right)=1+H_{2}$ by Lemma 3.5 , so assume $d^{L}\left(f_{1}, f_{2}\right) \leqslant 1$. By the structure of hops $d\left(v_{0}, x\right) \leqslant 1$ and $d\left(y, v_{2}\right) \leqslant 1$ for all vertices $x \prec f_{1}$ and $y \prec f_{2}$. Putting these inequalities together gives $d\left(v_{0}, v_{2}\right) \leqslant 1+1+1<2 H_{3}$. Since this contradicts the minimality of $P$ we conclude $d^{L}\left(f_{1}, f_{2}\right)=H_{2}$.

PART (3): By Lemma 3.5 we cannot have $d^{L}\left(S t^{L}\left(e_{1}\right), e_{2}\right)=1+H_{2}$, so assume $d^{L}\left(S t^{L}\left(e_{1}\right), e_{2}\right) \leqslant 1$. Let $\tilde{e}_{2}$ be the other transverse edge in the jump transverse to $e_{2}$. By the structure of jumps and the fact that $\operatorname{deg}\left(e_{1}\right) \leqslant 5$ we get $d\left(v_{0}, x\right) \leqslant 2$ for each vertex $x \in L k^{L}\left(e_{1}\right)$. Similarly, $\operatorname{deg}\left(\tilde{e}_{2}\right) \leqslant 5$ implies $d\left(y, v_{2}\right) \leqslant H_{3}$ for each vertex $y \prec e_{2}$. Combining these inequalities shows $d\left(v_{0}, v_{2}\right) \leqslant 2+1+H_{3}<2 J$. Since this contradicts the minimality of $P$, we have $d^{L}\left(S t^{L}\left(e_{1}\right), e_{2}\right)=H_{2}$.

In case (1), Corollary 3.3 implies $v_{2}$ is unique given $v_{0}$ and $v_{1}$. In cases (2) and (3), Lemma 3.5 shows that $L k\left(v_{1}\right)$ is an icosahedron in which $f_{2}$ and $e_{2}$ are uniquely determined by $f_{1}$ and $e_{1}$ respectively.

What about internal vertices adjacent to other combinations of edges, hops, and jumps within a minimal path? It turns out these cannot occur.

Lemma 4.2 A minimal path contains either all edges, all hops, or all jumps.

PROOF. Let $P$ be a minimal path with $P^{v}=\left(v_{0}, v_{1}, v_{2}\right)$, and note that $L=L k\left(v_{1}\right)$ is a positively curved surface by Lemma 3.7 .

CASE 1: Suppose $P^{l}=\left(1, H_{3}\right)$ with $f=\left[x_{0}, x_{1}, x_{2}\right]$ transverse to the hop. By Corollaries 3.2 and $3.3, d^{L}\left(v_{0}, f\right) \leqslant H_{2}$. Since $d\left(x_{i}, v_{2}\right)=1$ for each $x_{i}$, if $d^{L}\left(v_{0}, f\right) \leqslant 1$ we would get $d\left(v_{0}, v_{2}\right) \leqslant 2<1+H_{3}$, contradicting minimality of $P$. Therefore, $d^{L}\left(v_{0}, f\right)=H_{2}$ and a 2-hop exists in $L$ from $v_{0}$ to some $x_{i}$ (WLOG $x_{0}$ ). Let $e$ be transverse to this 2-hop.

Consider the 2-simplices $y_{1} *\left[x_{0}, x_{1}\right]$ and $y_{2} *\left[x_{0}, x_{2}\right]$ in $L$, each sharing an edge with $f$. Since the distinct edges $\left[y_{1}, x_{1}\right],\left[x_{1}, x_{2}\right],\left[x_{2}, y_{2}\right]$, and $e$ lie in $L k^{L}\left(x_{0}\right)$ and $\operatorname{deg}\left(x_{0}\right) \leqslant 5$ we know $y_{i} \prec e$ for some $y_{i}$ (WLOG $y_{1}$ ). This means a jump exists from $v_{0}$ to $v_{2}$ using the simplices $v_{0} *\left[v_{1}, y_{1}\right],\left[v_{1}, y_{1}\right] *\left[x_{0}, x_{1}\right]$, and $\left[x_{0}, x_{1}\right] * v_{2}$. Since $J<1+H_{3}$ this contradicts the minimality of $P$.

CASE 2: Suppose $P^{l}=(1, J)$ with $e_{1} \in L k\left(v_{1}\right)$ and $e_{2} \in L k\left(v_{2}\right)$ transverse to the jump. By Corollary 3.3 and Lemma 2.2 we have $d\left(v_{0}, e_{1}\right) \leqslant H_{3}$. Since $\operatorname{deg}\left(e_{2}\right) \leqslant 5$ we know $d\left(x, v_{2}\right) \leqslant H_{3}$ for each $x \prec e_{1}$. Combining these inequalities gives $d\left(v_{0}, v_{2}\right) \leqslant 2 H_{3}<1+J$ which contradicts the minimality of $P$.

CASE 3: Suppose $P^{l}=\left(H_{3}, J\right)$ with $f$ transverse to the hop, and $e_{1} \in L k\left(v_{1}\right)$ and $e_{2} \in L k\left(v_{2}\right)$ transverse to the jump. Let $f_{1}$ and $f_{2}$ be the two 2-simplices of $S t^{L}\left(e_{1}\right)$. By Lemma 3.5, for some $f_{i}$ we have $d^{L}\left(f, f_{i}\right) \leqslant 1$. Thus, by the structure of hops $d\left(v_{0}, S t^{L}\left(e_{1}\right)\right) \leqslant 2$. Using the structure of jumps and $\operatorname{deg}\left(e_{1}\right) \leqslant 5$ we get $d\left(x, v_{2}\right) \leqslant 2$ for each vertex $x \in \overline{S t^{L}\left(e_{1}\right)}$. Combining these inequalities gives $d\left(v_{0}, v_{2}\right) \leqslant 2+2<H_{3}+J$ which contradicts the minimality of $P$.

Lemma 2.6, Lemma 4.2 and the uniqueness given in Lemma 4.1 imply that, just as in the Riemannian setting, if a non-trivial minimal path can be extended to a longer minimal path then this extension is unique. 
Corollary 4.3 (Unique Extension) If two non-trivial minimal paths of equal length pass through the same first two simplices then the paths are identical.

Note that unique extension would not hold if our space of paths were defined using only edges. This illustrates an important advantage to expanding the space of paths to include those containing hops and jumps.

Now, we can begin to give arguments bounding the length of minimal paths in $M^{3}$. We start with paths containing only jumps.

Lemma 4.4 A minimal path contains at most two jumps.

Proof. Suppose $P$ contains three jumps, let $P^{v}=\left(v_{0}, v_{1}, v_{2}, v_{3}\right)$, and let $e_{1} \in L k\left(v_{2}\right)$ and $e_{2} \in L k\left(v_{3}\right)$ be transverse to the final jump. Since $\operatorname{deg}\left(e_{1}\right) \leqslant 5$, the structure of jumps implies $d\left(v_{2}, x\right) \leqslant H_{3}$ for some $x \prec e_{2}$ and therefore $d\left(v_{0}, x\right) \leqslant 2 J+H_{3}$. By the structure of jumps $d\left(x, v_{3}\right) \leqslant 1$, so that minimality of $P$ gives $d\left(v_{0}, x\right) \geqslant 3 J-1$. Combining these two inequalities implies $3 J-1 \leqslant d\left(v_{0}, x\right) \leqslant 2 J+H_{3}$. This is a contradiction because no minimal path allowed by Lemma 4.2 has length in this interval.

Our next lemma restricts the number of edges in a minimal path.

Lemma 4.5 Suppose $P_{x}$ is a five edge almost minimal path from $v$ to $w$ with first internal vertex $x \in L k(v)$. Then, each 2-simplex $f \in L k(v)$ with $x \prec f$ is transverse to the first hop in a three hop path $P_{f}$ from $v$ to $w$.

PROOF. (See Figure 6.) Suppose $P_{x}$ is an almost minimal five-edge path with $P_{x}^{v}=$ $\left(v, x, x_{1}, x_{2}, x_{3}, w\right)$. By Lemma $4.1(1)$, within the link of each internal vertex of $P_{x}$, the previous vertex and subsequent vertex along $P_{x}$ have maximum distance.

Any $f \in L k(v)$ with $x \prec f$ is transverse to a 3-hop from $v$ to a unique $w_{1} \in L k(x)$. This means a 2-hop in $L k(x)$ exists from $v$ to $w_{1}$. Using Corollary 3.4 (2) we know $w_{1} \in L k\left(x_{1}\right)$ so that (3) then provides a 2-hop in $L k\left(x_{1}\right)$ from $w_{1}$ to $x_{2}$ transverse to some edge $\tilde{e}$. Let $w_{2}$ be the vertex at the end of the unique 2-hop in $L k\left(x_{2}\right)$ which begins on $x_{1}$ and is transverse to $\tilde{e}$. Since a 2-hop from $x_{1}$ to $w_{2}$ exists in $L k\left(x_{2}\right)$, Corollary 3.4 (2) shows $w_{2} \in L k\left(x_{3}\right)$ and then Corollary 3.4 (3) gives a 2-hop in $L k\left(x_{3}\right)$ from $w_{2}$ to $w$. Thus, a 3-hop from $w_{2}$ to $w$ exists in $M^{3}$.

So far, we know vertices $w_{1}, x_{1}, x_{2}, w_{2}$ exist (in that order) within $\operatorname{Lk}(\tilde{e})$. If $\operatorname{deg}(\tilde{e}) \leqslant 4$ then $d\left(w_{1}, w_{2}\right) \leqslant 1$ which, along with $d\left(w_{2}, x_{3}\right) \leqslant 1$, would give $d\left(v, x_{3}\right) \leqslant H_{3}+2$. This would contradict the almost-minimality of $P$, so we conclude $\operatorname{deg}(\tilde{e})=5$. Therefore a 3-hop exists from $w_{1}$ to $w_{2}$. This means a three hop path $P_{f}$ with $P_{f}^{v}=\left(v, w_{1}, w_{2}, w\right)$ exists in $M^{3}$ with the desired properties.

Since $3 H_{3}<5$ we get:

Corollary 4.6 A minimal path contains at most four edges.

Next, we bound the number of hops in a minimal path using: 


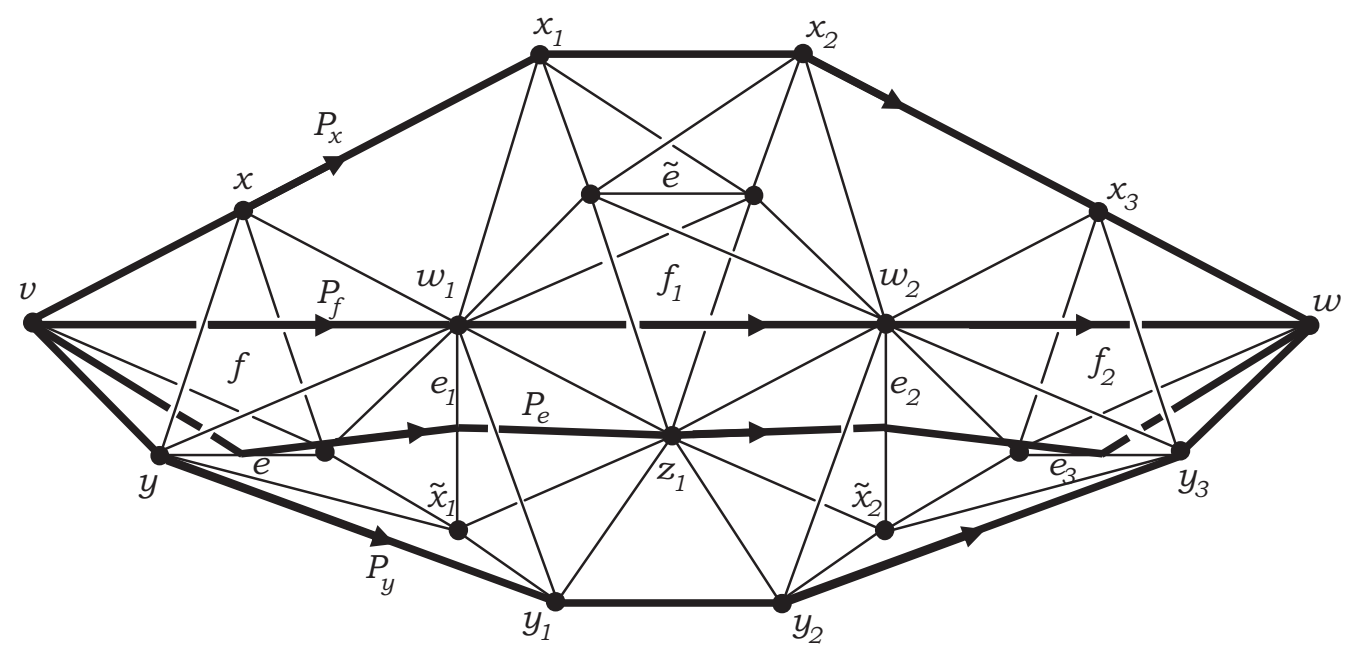

Figure 6: Nearby paths from $v$ to $w$

Lemma 4.7 Suppose $P_{f}$ is a three hop almost minimal path from $v$ to $w$ with the 2simplex $f \in L k(v)$ transverse to the first hop. Then, each edge $e \prec f$ is transverse to the first jump in a two jump path $P_{e}$ from $v$ to $w$.

PROOF. (See Figure 6.) Let $P_{f}^{v}=\left(v, w_{1}, w_{2}, w\right)$ with $f_{1}$ and $f_{2}$ transverse to the second and third hops respectively. By Lemma $4.1(2)$ we know the $L k\left(w_{i}\right)$ are icosahedra in which $d\left(f, f_{1}\right)=H_{2}$ and $d\left(f_{1}, f_{2}\right)=H_{2}$. By Lemma 3.6 (1) applied to $L k\left(w_{1}\right)$, each $e \prec f$ corresponds to unique vertices $\tilde{x}_{1} \in L k(e) \cap L k\left(w_{1}\right)$ and $z_{1} \prec f_{1}$ such that $\left[\tilde{x}_{1}, z_{1}\right]$ is an edge. This means we can construct a jump from $v$ to $z_{1}$ using $v * e, e *\left[\tilde{x}_{1}, w_{1}\right]$, and $\left[\tilde{x}_{1}, w_{1}\right] * z_{1}$. Now, since $z_{1} \prec f_{1}$, Lemma $3.6(1)$ applied to $L k\left(w_{2}\right)$ gives a unique edge $e_{3} \prec f_{2}$ and vertex $\tilde{x}_{2} \in L k\left(e_{3}\right) \cap L k\left(w_{2}\right)$ so that $\left[z_{1}, \tilde{x}_{2}\right]$ is an edge. Thus we can construct a jump from $z_{1}$ to $w$ using $z_{1} *\left[\tilde{x}_{2}, w_{2}\right],\left[\tilde{x}_{2}, w_{2}\right] * e_{3}$, and $e_{3} * w$. This completes the desired path $P_{e}$.

Since $2 \mathrm{~J}<3 \mathrm{H}_{3}$ we have the desired corollary:

Corollary 4.8 A minimal path contains at most two hops.

Lemma 4.4 and Corollaries 4.6 and 4.8 together show that $\operatorname{Diam}\left(M^{3}\right) \leqslant 2 J$. In fact, we have this stronger result:

Corollary $4.9 d(v, w) \in\left\{0,1, H_{3}, 2, J, 3,2 H_{3}, 4,2 J\right\}$ for any vertices $v, w \in M^{3}$.

Thus, since a hop can spanned by two edges and a jump by three, $d(v, w) \neq 2 J$ implies $d_{1}(v, w) \leqslant 4$. Our next result shows that $d_{1}(v, w)=5$ for $d(v, w)=2 J$, completing the proof of Theorem 1.1 for $n=3$.

Lemma 4.10 Suppose $P_{e}$ is a two jump minimal path from $v$ to $w$ and the edge $e \in L k(v)$ is transverse to the first jump. Then, each vertex $y \prec e$ is the first internal vertex of a five edge path $P_{y}$ from $v$ to $w$. 
PRoOF. (See Figure 6.) Suppose $P_{e}^{v}=\left(v, z_{1}, w\right)$ and let $e_{1} \in L k\left(z_{1}\right)$ be transverse to the first jump, and $e_{2} \in L k\left(z_{1}\right), e_{3} \in L k(w)$ transverse to the second. Since the first jump is minimal we must have $\operatorname{deg}\left(e_{1}\right)=5$, giving a unique vertex $y_{1} \in \operatorname{Lk}\left(e_{1}\right) \cap \operatorname{Lk}\left(z_{1}\right)$ such that $\left[y, y_{1}\right]$ an edge.

By Lemma $4.1(3), L k\left(z_{1}\right)$ is an icosahedron and $d^{L k\left(z_{1}\right)}\left(S t\left(e_{1}\right), e_{2}\right)=H_{2}$. Therefore Lemma 3.6 (2) associates to $y_{1}$ a unique $y_{2} \in L k\left(e_{2}\right) \cap L k\left(z_{1}\right)$ where $\left[y_{1}, y_{2}\right]$ is an edge. Since the second jump is minimal $\operatorname{deg}\left(e_{2}\right)=5$ and we have a unique vertex $y_{3} \prec e_{3}$ such that $\left[y_{2}, y_{3}\right]$ is an edge. Since the structure jumps gives an edge $\left[y_{3}, w\right]$ we have finished constructing our $P_{y}$.

\section{$5 \quad$ Structure of an $M^{3}$ with Maximum Diameter}

Throughout this section we suppose $d_{1}(v, w)=5$ for some vertices $v, w \in M^{3}$. Since hops can be traversed by two edges, and jumps by three, $d(v, w)=2 J$ by Corollary 4.9. So, let $P$ be a minimal path from $v$ to $w$ realizing this distance. Repeatedly applying Lemmas 4.10, 4.7 and 4.5 allows us to generate paths $P_{\sigma}$ from $v$ to $w$ passing through each simplex $\sigma \in L k(v)$.

Lemma 5.1 $P_{\sigma}$ is minimal if $\sigma$ is an edge and almost minimal otherwise.

PROOF. If $\sigma$ is an edge then $P_{\sigma}$ has length $2 J$ and must be minimal since $d(v, w)=2 J$. Suppose $\sigma$ is a 2-simplex. If $P_{\sigma}$ is not almost minimal then some two-hop subpath of $P_{\sigma}$ is not minimal. By Corollary 4.9, this would imply $d(v, w) \leqslant 3+H_{3}<2 J$ contradicting $d(v, w)=2 J$. Similarly, if $\sigma$ is a vertex and $P_{\sigma}$ is not almost minimal, then some four-edge subpath of $P_{\sigma}$ is not minimal. By Corollary 4.9 this would imply $d(v, w) \leqslant 2 H_{3}+1<2 J$ which is again a contradiction.

The above lemma and Corollary 4.3 imply that each path $P_{\sigma}$ is uniquely determined by $\sigma$. Since every vertex in $M$ lies on some minimal path beginning on $v$, this provides a convenient way to refer to vertices in $M$.

Definition 5.2 We say a vertex $x \in M$ has coordinates $\langle\sigma, i\rangle \in L k(v) \times \mathbb{Z}$ if $x$ is the $(i+1)$-st vertex along $P_{\sigma}$.

These coordinates are very similar to geodesic normal coordinates in the Riemannian setting. Just as in the case of a standard Riemannian sphere, except for the "antipodal" points $v$ and $w$, each vertex in $M$ has unique coordinates.

Corollary 5.3 Each vertex in $M \backslash\{v, w\}$ is an internal vertex of a unique $P_{\sigma}$ and therefore has unique coordinates.

Now we will explicitly describe the structure of $M$ near each vertex except $v$ and $w$. Specifically, for each internal vertex $\langle\sigma, i\rangle$ along each $P_{\sigma}$, we will list the simplices in $L k(\langle\sigma, i\rangle)$ and give explicit coordinates for their vertices. Essentially, all the information 
needed is already implicit in the proofs of Lemmas 4.5, 4.7 and 4.10. Since Figure 6 depicts the face relations between the various simplices which appear in these proofs, we encourage the reader to refer to this figure while reading the proofs of the next three lemmas.

We begin by looking at the internal vertices along $P_{x}$ where $x$ is a vertex.

Lemma 5.4 Consider a vertex $\langle x, i\rangle$ where $x \in L k(v)$ is a vertex and $i=1, \ldots, 4$. The structure of $L k(\langle x, i\rangle)$ is as shown in Figure 7.

PROOF. Lemma 4.1 implies each 2-sphere $L k(\langle x, i\rangle)$ has maximum diameter with vertices $\langle x, i-1\rangle$ and $\langle x, i+1\rangle$ at maximum distance. So, Corollary 3.4 shows that for $i=1, \ldots, 4$, within $L k(\langle x, i\rangle)$ we have $\operatorname{deg}(\langle x, i-1\rangle)=\operatorname{deg}(\langle x, i+1\rangle)$. (Let $k$ denote this degree.) This means the $L k(\langle x, i\rangle)$ for $i=1, \ldots, 4$ are all isomorphic, as shown in Figure 7 .

Lemma 4.1 fixes the relative positions of the vertices $\langle x, 0\rangle, \ldots,\langle x, 5\rangle$ in the table. Clearly, if the vertices $x_{i} \in L k(v)$ for $i=1, \ldots k$ are as shown in the lower drawing, then the $\left\langle x_{i}, 1\right\rangle$ must be placed as they are in the table. The proof of Lemma 4.5 applied to $P_{x}$ fixes the positions of the vertices $\left\langle f_{i}, 1\right\rangle$ and $\left\langle f_{i}, 2\right\rangle$ for $i=1, \ldots, k$. Finally, the proof of Lemma 4.10 applied to each $P_{e_{i}}$ fixes the placement of the vertices $\left\langle e_{i}, 1\right\rangle$ for $i=1, \ldots, k$.

Next, we examine the internal vertices along $P_{f}$ where $f$ is an 2 -simplex.

Lemma 5.5 Consider a vertex $\langle f, i\rangle$ where $f \in L k(v)$ is a 2-simplex and $i=1,2$. The structure of $L k(\langle f, i\rangle)$ is as shown in Figure 8.

PROOF. Lemma 4.1 implies $L k(\langle f, 1\rangle)$ and $L k(\langle f, 2\rangle)$ are icosahedra as shown in Figure 8. Clearly, if the vertices $x_{1}, x_{2}$ and $x_{3}$ are as shown in the lower drawing, then the relative placement of $\left\langle x_{1}, 1\right\rangle,\left\langle x_{2}, 1\right\rangle$ and $\left\langle x_{3}, 1\right\rangle$ must be as shown in the table. The proof of Lemma 4.7 applied to $P_{f}$ fixes the position of the vertices $\left\langle e_{i}, 1\right\rangle$ for $i=1,2,3$. The proof of Lemma 4.10 applied to the paths $P_{e_{i}}$ fixes the positions of vertices $\left\langle x_{i}, j\right\rangle$ for $i=1,2,3$ and $j=2,3,4$. Finally, the proof of Lemma 4.5 applied to the paths $P_{x_{i}}$ fixes the positions of the vertices $\left\langle f_{i}, j\right\rangle$ for $i=1,2,3$ and $j=1,2$.

Finally, we examine the internal vertex of $P_{e}$ where $e$ is an edge.

Lemma 5.6 Consider a vertex $\langle e, 1\rangle$ where $e \in L k(v)$ is an edge. The structure of Lk $(\langle e, 1\rangle)$ is as shown in Figure 9.

PROOF. Lemma 4.1 implies $L k(\langle e, 1\rangle)$ is an icosahedron as shown in Figure 8. Applying the proof of Lemma 4.7 to $P_{f_{1}}$ and $P_{f_{2}}$ fixes the relative positions of the vertices $\left\langle f_{i}, j\right\rangle$ for $i=1,2$ and $j=1,2$. Applying the proof of Lemma 4.7 again to $P_{f_{1}}$ and $P_{f_{2}}$ fixes the positions of the $e_{i j}$ for $i=1,2$ and $j=1,2$. Finally, applying the proof of Lemma 4.10 to $P_{e}$ fixes the positions of the vertices $\left\langle x_{i}, j\right\rangle$ for $i=1,2$ and $j=2,3$.

Now we are ready to proceed with the proof of Theorem 1.2 part (2) for $n=3$. For the reader's convenience we restate this result. 


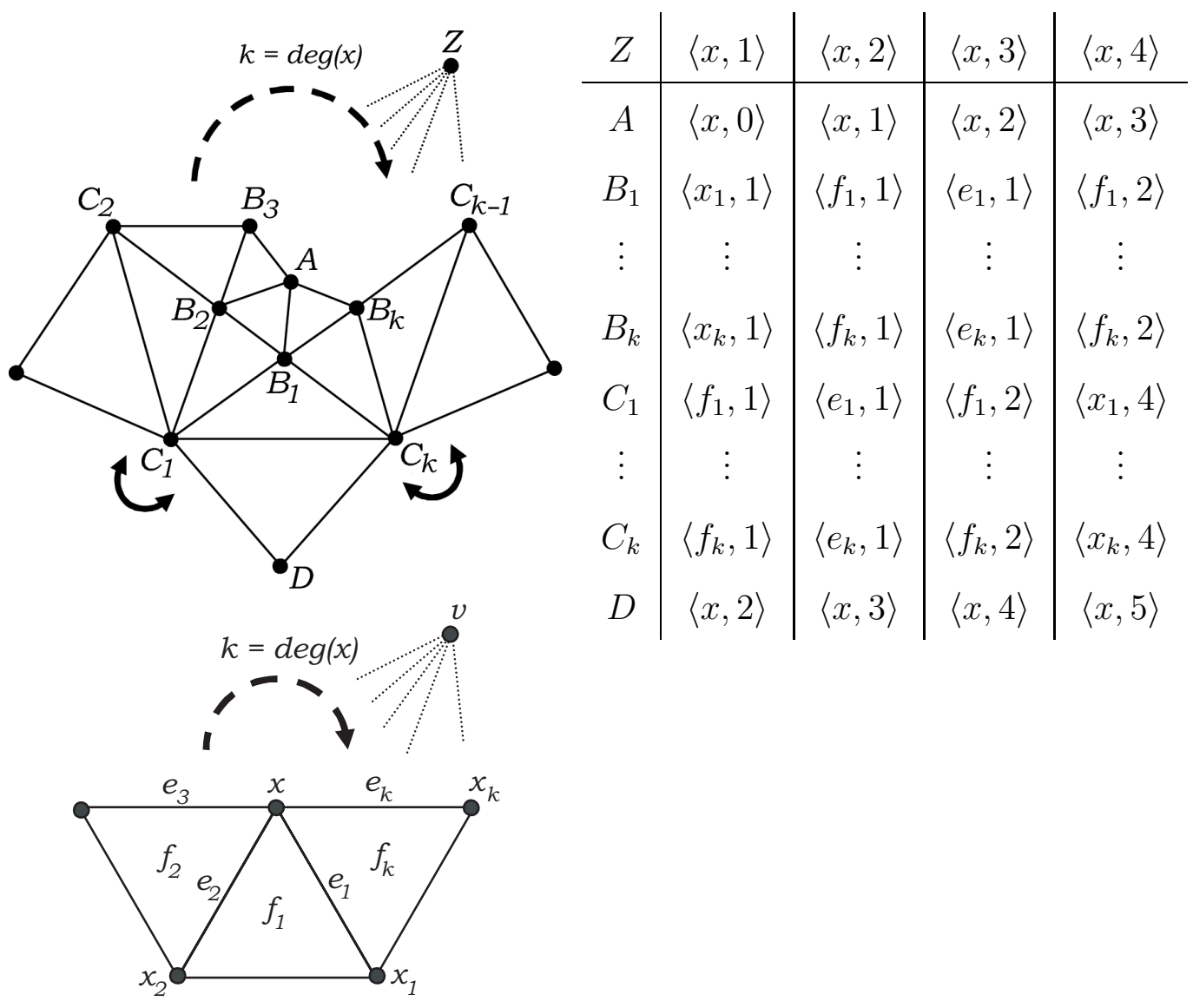

Figure 7: (See Lemma 5.4) This figure specifies the structure of $L k(\langle x, i\rangle)$ where $x$ is a vertex in $L k(v)$ with degree $k$ and $i=1, \ldots, 4$. The coordinates of each vertex in $L k(\langle x, i\rangle)$ are listed in the table as entries in the column headed $\langle x, i\rangle$. The other simplices in $L k(\langle x, i\rangle)$ are as shown in the top left drawing. In this drawing, each vertex has a label $Z, A, \ldots, D$ which corresponds to a row in the table. The simplices $\sigma \in L k(v)$ which appear as coordinates $\langle\sigma, j\rangle$ in the table are located "near" $x$ in $L k(v)$. The specific face-relations between $x$ and these "nearby" $\sigma$ are indicated in the lower left drawing. In this way, the table and the two drawings completely specify the structure of $L k(\langle x, i\rangle)$ for $i=1, \ldots, 4$. 


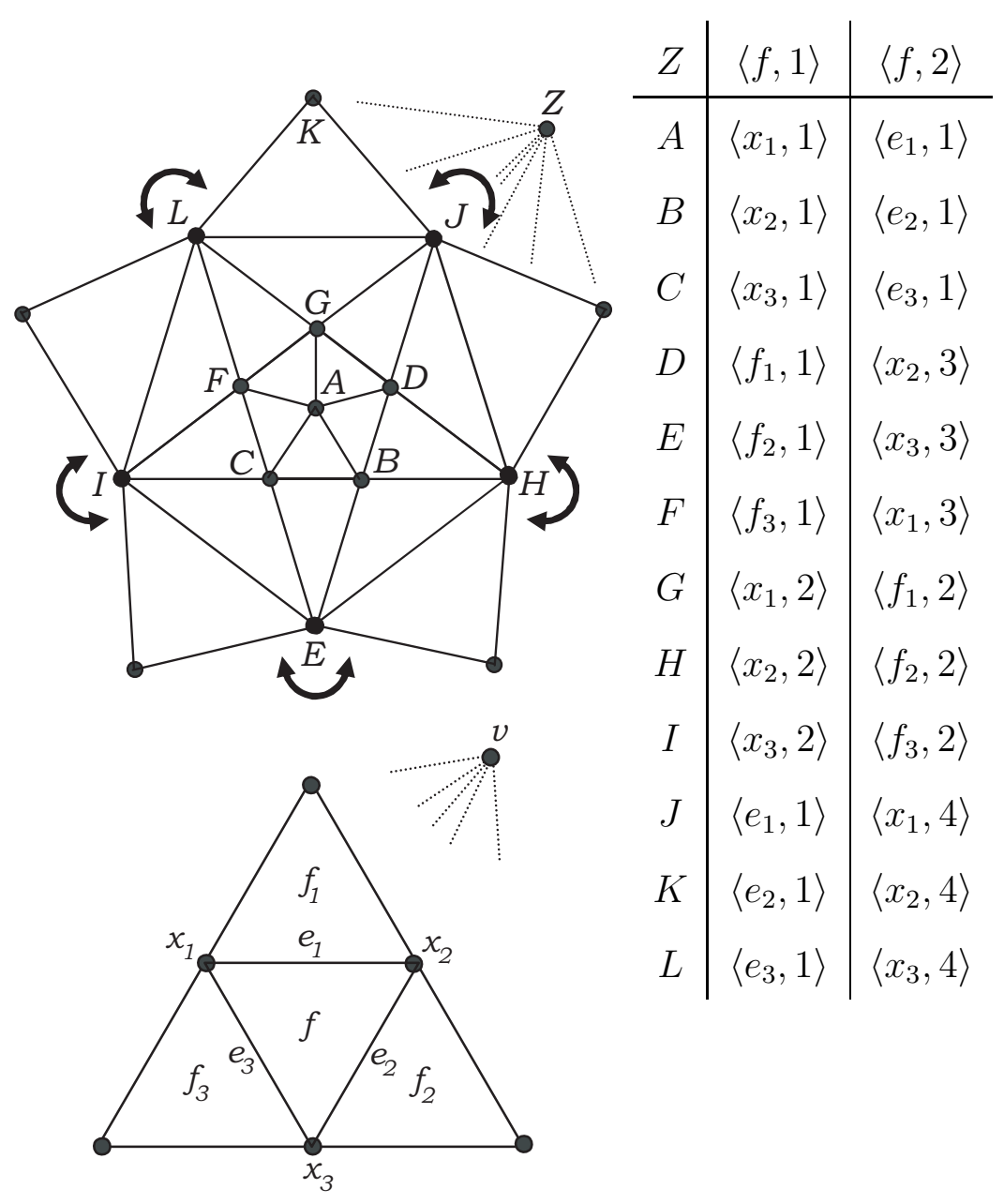

Figure 8: (See Lemma 5.5) This figure specifies the structure of $L k(\langle f, i\rangle)$ where $f$ is a 2 -simplex in $L k(v)$ and $i=1,2$. The coordinates of each vertex in $L k(\langle f, i\rangle)$ are listed in the table as entries in the column headed $\langle f, i\rangle$. The other simplices in $L k(\langle f, i\rangle)$ are as shown in the top left drawing. In this drawing, each vertex has a label $Z, A, \ldots, L$ which corresponds to a row in the table. The simplices $\sigma \in L k(v)$ which appear as coordinates $\langle\sigma, j\rangle$ in the table are located "near" $f$ in $L k(v)$. The specific face-relations between $f$ and these "nearby" $\sigma$ are indicated in the lower left drawing. In this way, the table and the two drawings completely specify the structure of $L k(\langle f, i\rangle)$ for $i=1,2$. 


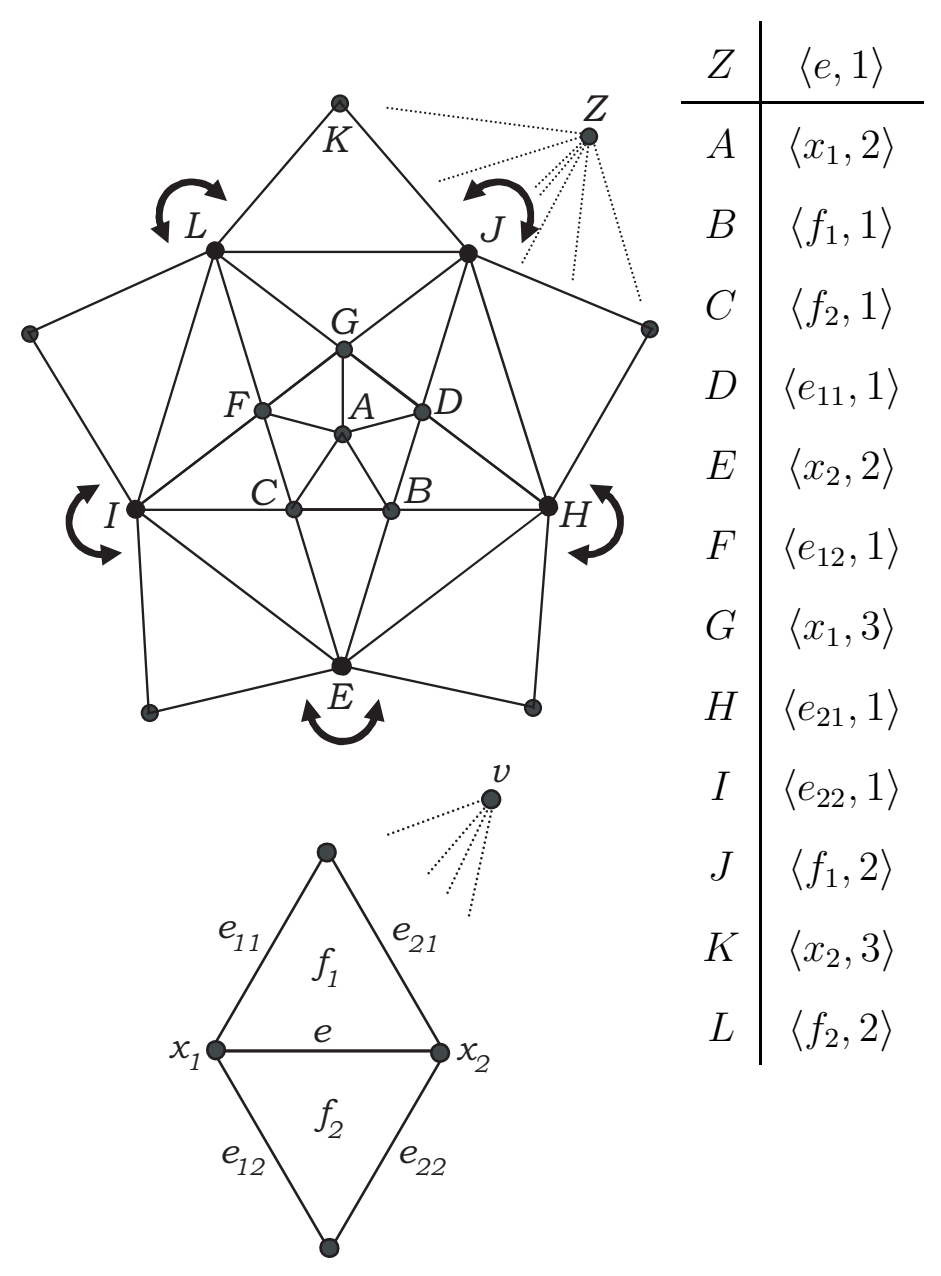

Figure 9: (See Lemma 5.6) This figure specifies the structure of $L k(\langle e, 1\rangle)$ where $e$ is an edge in $L k(v)$. The coordinates of each vertex in $L k(\langle e, 1\rangle)$ are listed in the table as entries in the column headed $\langle e, 1\rangle$. The other simplices in $L k(\langle e, 1\rangle)$ are as shown in the top left drawing. In this drawing, each vertex has a label $Z, A, \ldots, L$ which corresponds to a row in the table. The simplices $\sigma \in L k(v)$ which appear as coordinates $\langle\sigma, j\rangle$ in the table are located "near" $e$ in $L k(v)$. The specific face-relations between $e$ and these "nearby" $\sigma$ are indicated in the lower left drawing. In this way, the table and the two drawings completely specify the structure of $L k(\langle e, 1\rangle)$. 
Theorem 1.2 (Part 2) Let $M$ be a positively curved combinatorial 3-manifold with vertices $v, w$ at edge-distance five. If $M^{\prime}$ is another positively curved 3-manifold with vertices $v^{\prime}, w^{\prime}$ at edge-distance five and there exists a simplicial isomorphism $\Psi: L k(v) \cong$ $L k\left(v^{\prime}\right)$ then $\Psi$ extends to a simplicial isomorphism $M \cong M^{\prime}$.

PROOF. Let $\Psi: L k(v) \rightarrow L k\left(v^{\prime}\right)$ be the given simplicial isomorphism. We have seen that each vertex in $M$ has coordinates in $L k(v) \times \mathbb{Z}$. Similarly, each vertex in $M^{\prime}$ has coordinates in $L k\left(v^{\prime}\right) \times \mathbb{Z}$. We can therefore extend $\Psi$ as follows, using coordinates to refer to vertices.

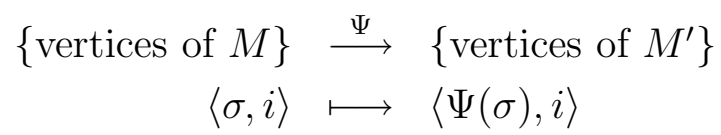

Corollary 5.3 implies this is a well-defined bijection which agrees with the original $\Psi$ on the vertices of $L k(v)$. We can extend $\Psi$ to an isomorphism $\Psi: M \rightarrow M^{\prime}$ by sending $\tau=\left[x_{0}, \ldots, x_{k}\right] \mapsto\left[\Psi\left(x_{0}\right), \ldots, \Psi\left(x_{k}\right)\right]=\Psi(\tau)$ provided that $\Psi(\tau) \in M^{\prime}$ for each $\tau \in M$.

Since $M$ is a combinatorial 3-manifold, it is enough to check that $\Psi(\tau) \in M^{\prime}$ when $\tau=\left[x_{0}, \ldots, x_{3}\right]$ is an arbitrary 3-simplex in $M$. By Corollary 5.3, some $x_{i}$ (WLOG $x_{0}$ ) is an internal vertex of some $P_{\sigma}$ and has unique coordinates $\langle\sigma, k\rangle$ where $\sigma \in L k(v)$ and $k \geqslant 1$. Now, depending on the dimension of $\sigma$, we can apply either Lemma 5.4, 5.5 or 5.6 at the vertex $x_{0}$. This uniquely identifies the vertices $x_{1}, x_{2}$ and $x_{3}$ as having coordinates $\left\langle\sigma_{1}, k_{1}\right\rangle,\left\langle\sigma_{2}, k_{2}\right\rangle$ and $\left\langle\sigma_{3}, k_{3}\right\rangle$ respectively.

By the definition of $\Psi$ we know $\Psi\left(x_{0}\right) \in M^{\prime}$ has coordinates $\langle\Psi(\sigma), k\rangle$ and $\Psi\left(x_{i}\right)$ has coordinates $\left\langle\Psi\left(\sigma_{i}\right), k_{i}\right\rangle$ for $i=1,2,3$. Since $\Psi$ is a simplicial isomorphism, $\sigma$ and $\Psi(\sigma)$ are either both 2-simplices, both edges, or both vertices of the same degree (within $L k(v)$ and $L k\left(v^{\prime}\right)$ respectively). Moreover, whatever the relative positions in $L k(v)$ between $\sigma$ and the $\sigma_{i}$, the corresponding simplices $\Psi(\sigma)$ and $\Psi\left(\sigma_{i}\right)$ must have the same relative positions in $L k\left(v^{\prime}\right)$. Now, we apply either Lemma $5.4,5.5$ or 5.6 at the vertex $\langle\Psi(\sigma), k\rangle$. This implies that if the vertices $\left\langle\sigma_{i}, k_{i}\right\rangle$ form a 2 -simplex in $L k(\langle\sigma, k\rangle)$ then the corresponding vertices $\left\langle\Psi\left(\sigma_{i}\right), k_{i}\right\rangle$ must form a 2-simplex in $L k(\langle\Psi(\sigma), k\rangle)$. Therefore, $\Psi(\tau)=\left[\Psi\left(x_{0}\right), \Psi\left(x_{1}\right), \Psi\left(x_{2}\right), \Psi\left(x_{3}\right)\right]=\left[\langle\Psi(\sigma), k\rangle,\left\langle\Psi\left(\sigma_{1}\right), k_{1}\right\rangle,\left\langle\Psi\left(\sigma_{2}\right), k_{2}\right\rangle,\left\langle\Psi\left(\sigma_{3}\right), k_{3}\right\rangle\right]$ is a 3 -simplex in $M^{\prime}$ as desired.

We finish the section with the proof of part (3) of Theorem 1.2 which we restate here for the reader's convenience.

Theorem 1.2 (Part 3) For each 2-sphere $L$ with vertices of degree at most five, we explicitly construct a positively curved $M^{3}$ with vertices $v$ and $w$ at edge-distance five and $L k(v)=L$.

PROOF. Suppose $L$ is a positively curved combinatorial 2-sphere. We wish to find a positively curved 3-manifold $M$ in which $d_{1}(v, w)=5$ and $L k(v)=L$. To do this we simply use Figures 7, 8 and 9 to define all the new simplices we need for $M$, based on the structure of $L$. There are four things to check. First, this procedure must consistently define a simplicial complex. That is, we must check that none of the information in Figures 7, 8 and 9 is contradictory when the portions of $L k(v)$ shown in the lower drawing 


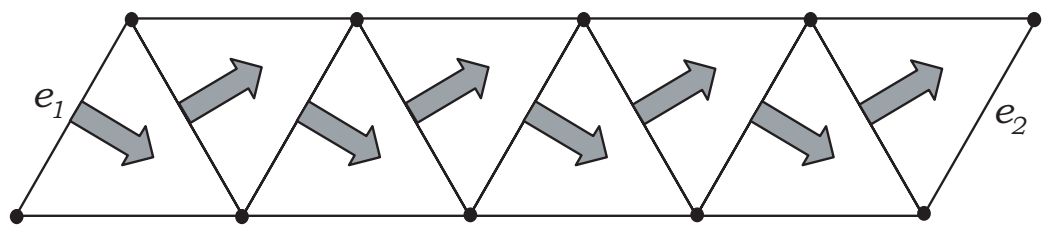

Figure 10: Discrete Morse path from $e_{1}$ to $e_{2}$

in each figure overlap. Second, we need to verify that $M$ is a combinatorial 3-manifold by showing that the link of every vertex in $M$ is a 2 -sphere. Third, we must check that $M$ is positively curved by demonstrating each edge in $M$ has degree at most five. Finally, we should verify that the vertices $v$ and $w$ have edge-distance five. This can be done by computing the edge-distance to $v$ for each vertex in Figures 7, 8 and 9, starting at $v$ and working towards $w$. These verifications are tedious, but all of the relevant information is contained in Figures 7, 8 and 9. Once we are done, we see that $M$ is indeed the desired combinatorial 3-manifold.

\section{Forman's Discrete Morse Theory}

It remains to show that any maximum diameter $M$ is a sphere. To do this, we use Discrete Morse Theory. This is a beautiful discrete version of classical Morse theory developed by Robin Forman in [5]. It requires very little structure in the underlying space. Here, we need only assume that $M$ is a finite simplicial complex.

Definition 6.1 A discrete Morse arrow is a codimension-1 pair $\sigma^{k-1} \prec \tau^{k}$ of simplices from $M$. We depict this by placing an arrow pointing from $\sigma$ into $\tau$.

These arrows can be put together to form paths.

Definition 6.2 A discrete Morse path is a list of simplices $\sigma_{1}, \ldots, \sigma_{k}$ for which, alternately

1. $\sigma_{i} \succ \sigma_{i+1}$ are a codimension-1 pair but not part of an arrow, and

2. $\sigma_{i} \prec \sigma_{i+1}$ follows a discrete Morse arrow.

See Figure 10 for an illustration of a discrete morse path.

Analogous to the classical notion of a Morse function on a smooth manifold is the following:

Definition 6.3 A set $\mathbb{F}$ of discrete Morse arrows on $M$ is called a discrete Morse function if

1. no simplex in $M$ appears in more than one arrow, and 
2. no discrete Morse path using the arrows in $\mathbb{F}$ forms a loop.

A simplex not part of any arrow is called critical.

The fundamental theorem in Discrete Morse Theory is then:

Theorem 6.4 (Forman) If $\mathbb{F}$ is a discrete Morse function on $M$ with $c_{i}$ critical $i$ simplices then $M$ is (simple)-homotopy equivalent to a $C W$-complex containing $c_{i} i$-cells.

We will need only one other result from Forman's work. It follows from Theorem 6.4 and Whitehead's Theorem of Regular Neighborhoods.

Theorem 6.5 (Forman) If a combinatorial manifold with boundary admits a discrete Morse function whose only critical simplex is a vertex then that manifold is homeomorphic to a ball.

\subsection{Constructing Discrete Morse Functions}

We begin with an arbitrary function

$$
g:\{\text { vertices in } M\} \rightarrow \mathcal{O}
$$

assigning a value in some totally ordered set $\mathcal{O}$ to each vertex of $M$. We call this function our vertex ordering function. From $g$ we will construct a Discrete Morse Function on $M$ whose arrows point more-or-less in the direction of decreasing $g$.

Now for a few definitions and some notation. We will need a convenient way to denote the maximum of $g$ on a simplex. So, let

$$
\bar{g}(\sigma)=\max _{x \preceq \sigma} g(x) .
$$

We also define notation for the number of vertices in a simplex which attain the maximum. That is, we let

$$
g^{\#}(\sigma)=\mid\{x \mid x \prec \sigma \text { is a vertex with } g(x)=\bar{g}(\sigma)\} \mid .
$$

Next, we define sets describing the local behavior of $g$.

Definition 6.6 Let $g$ be any vertex ordering function on $M$. The constant set of $M$ is given by

$$
C_{g}(M)=\{\sigma \in M \mid g(x)=\bar{g}(\sigma) \text { for every vertex } x \prec \sigma\} .
$$

For any simplex $\sigma \in C_{g}(M)$ we define the following two sets. The descending link of $\sigma$ is the subcomplex of $L k(\sigma)$ given by

$$
L k_{g}^{-}(\sigma)=\{\tau \in L k(\sigma) \mid \bar{g}(\tau)<\bar{g}(\sigma)\} .
$$

The descending star of $\sigma$ is the subset of $S t(\sigma)$ given by

$$
S t_{g}^{-}(\sigma)=\{\sigma\} \cup\left\{\sigma * \tau \mid \tau \in L k_{g}^{-}(\sigma)\right\} .
$$

When the identity of $g$ is clear, we will usually omit the " $g$ " from the above notations. 
Our first lemma shows that any simplicial complex is the disjoint union of its descending stars.

Lemma 6.7 For any $M$ and $g$ we have $M=\bigsqcup_{\sigma \in C_{g}(M)} S t_{g}^{-}(\sigma)$.

Proof. Consider $\rho \in M$. If $\rho \in C(M)$ then $\rho \in S t^{-}(\rho)$ by definition. If $\rho \in M \backslash C(M)$ then $\rho \in S t^{-}(\sigma)$ where the vertex set of $\sigma$ contains exactly the vertices of $\rho$ with largest $g$-value. Since $\sigma$ is uniquely determined by $\rho$, the descending stars $S t^{-}(\sigma)$ are disjoint.

Starting from a set of discrete Morse arrows on a descending link we can construct sets of arrows on the corresponding descending star.

Definition 6.8 Suppose $\sigma$ is in $C(M)$. We say that a set of discrete Morse arrows $\mathbb{F}^{*}$ on $S t^{-}(\sigma)$ is induced by a set of discrete Morse arrows $\mathbb{F}$ on $L k^{-}(\sigma)$ if $\mathbb{F}^{*}$ is constructed in the following manner.

1. If $L k^{-}(\sigma)$ contains a vertex $v$ which is not part of an arrow in $\mathbb{F}$ then we place an arrow in $\mathbb{F}^{*}$ from $\sigma$ to $\sigma * v$.

2. We place an arrow in $\mathbb{F}^{*}$ from $\sigma * \tau_{1}$ to $\sigma * \tau_{2}$ if and only if there is an arrow in $\mathbb{F}$ from $\tau_{1}$ to $\tau_{2}$.

Here is an important fact following immediately from this definition.

Corollary 6.9 Suppose $\mathbb{F}$ is a discrete Morse function on $L k^{-}(\sigma)$ whose only critical simplex is a vertex. Let $\mathbb{F}^{*}$ be a set of discrete Morse arrows induced by $\mathbb{F}$. Then, every simplex in $S t^{-}(\sigma)$ is part of an arrow in $\mathbb{F}^{*}$.

These induced sets of arrows are useful because of the following result.

Lemma 6.10 Suppose that for each $\sigma \in C(M)$ we have a discrete Morse function $\mathbb{F}_{\sigma}$ on $L k^{-}(\sigma)$. Then, the disjoint union

$$
\mathbb{F}=\bigsqcup_{\sigma \in C(M)} \mathbb{F}_{\sigma}^{*}
$$

forms a discrete Morse function on $M$ for any set of induced $\mathbb{F}_{\sigma}^{*}$.

PROOF. Since the $S t^{-}(\sigma)$ are disjoint, no simplex in $M$ appears in more than one arrow from $\mathbb{F}$. Suppose some discrete Morse path formed a loop. That is, suppose $P=$ $\left(\alpha_{1}, \ldots, \alpha_{k}\right)$ is a discrete Morse path in $M$ formed from arrows in $\mathbb{F}$ with $\alpha_{1}=\alpha_{k}$ and $k>2$.

Let the function $\mathcal{G}: M \rightarrow \mathcal{O} \times \mathbb{Z}$ be given by $\mathcal{G}(\tau)=\left(\bar{g}(\tau), g^{\#}(\tau)\right)$. Values of $\mathcal{G}$ can be compared using the standard dictionary order on $\mathcal{O} \times \mathbb{Z}$. It follows immediately from this definition that $\mathcal{G}$ is constant on each descending star and $\mathcal{G}(\gamma) \leqslant \mathcal{G}(\beta)$ for any simplices $\gamma \prec \beta$. 
Consider an arbitrary pair $\alpha_{i} \prec \alpha_{i+1}$ of codimension-1 simplices along $P$ paired into an arrow by $\mathbb{F}$ and let $\mathbb{F}_{\sigma}^{*}$ be the induced set containing this arrow. Since $\alpha_{i}$ and $\alpha_{i+1}$ are both in $S t^{-}(\sigma)$ we have $\mathcal{G}(\sigma)=\mathcal{G}\left(\alpha_{i}\right)=\mathcal{G}\left(\alpha_{i+1}\right)$. Next, consider what happens as we move from $\alpha_{i+1}$ to $\alpha_{i+2}$. Because $P$ is a discrete Morse path, $\alpha_{i+2}$ must be a codimension-1 face of $\alpha_{i+1}$ and the pair $\alpha_{i+1} \succ \alpha_{i+2}$ cannot be part of an arrow.

By the definition of the induced set of arrows $\mathbb{F}_{\sigma}^{*}$ there are two possibilities for $\alpha_{i}$ and $\alpha_{i+1}$.

CASE 1: Suppose $\alpha_{i}=\sigma$ and $\alpha_{i+1}=\sigma * v$ for a vertex $v \in L k^{-}(\sigma)$ not part of any arrow in $\mathbb{F}_{\sigma}$. Since $\sigma$ and $\sigma * v$ are part of an arrow in $\mathbb{F}_{\sigma}^{*}$ we know $\alpha_{i+2}$ cannot be $\sigma$. Any other codimension- 1 face of $\sigma * v$ has $g^{\#}$-value strictly smaller than $g^{\#}\left(\alpha_{i+1}\right)$. So $\mathcal{G}\left(\alpha_{i+1}\right)>\mathcal{G}\left(\alpha_{i+2}\right)$ and the path must leave $S t^{-}(\sigma)$.

CASE 2: Suppose $\alpha_{i}=\sigma * \tau_{i}$ and $\alpha_{i+1}=\sigma * \tau_{i+1}$ for $\tau_{i}, \tau_{i+1} \in L k^{-}(\sigma)$ paired by an arrow from $\mathbb{F}_{\sigma}$. If $\sigma$ is a face of $\alpha_{i+2}$ then $P$ stays in $S t^{-}(\sigma)$ and $\mathcal{G}\left(\alpha_{i+1}\right)=\mathcal{G}\left(\alpha_{i+2}\right)$. If $\sigma$ is not a face of $\alpha_{i+2}$ then $g^{\#}\left(\alpha_{i+1}\right)>g^{\#}\left(\alpha_{i+2}\right)$ which implies $\mathcal{G}\left(\alpha_{i+1}\right)>\mathcal{G}\left(\alpha_{i+2}\right)$ and tells us the path must leave $S t^{-}(\sigma)$.

Thus, $\mathcal{G}\left(\alpha_{i}\right)$ is a non-increasing function of $i$ for $0 \leqslant i \leqslant k$. Since $P$ forms a loop, $\mathcal{G}$ must be constant along $P$. By the arguments above, this implies two things. First, all the arrows $\alpha_{i}=\sigma * \tau_{i} \prec \sigma * \tau_{i+1}=\alpha_{i+1}$ along $P$ come from arrows $\tau_{i} \prec \tau_{i+1}$ in a single $\mathbb{F}_{\sigma}$. Second, each non-arrow transition $\alpha_{i+1}=\sigma * \tau_{i+1} \succ \sigma * \tau_{i+2}=\alpha_{i+2}$ along $P$ corresponds to a pair $\tau_{i+1} \succ \tau_{i+2}$ in $L k^{-}(\sigma)$ not connected by an arrow in $\mathbb{F}_{\sigma}$. This means the sequence $P^{-}=\left(\tau_{1}, \ldots, \tau_{k}\right)$ is a discrete Morse path on $L k^{-}(\sigma)$. Finally, since $P$ forms a loop so does $P^{-}$, contradicting the assumption that $\mathbb{F}_{\sigma}$ was a discrete Morse function.

The advantage to constructing discrete Morse functions on $M$ in this way is that we may independently choose the $\mathbb{F}_{\sigma}$ on each descending link $L k^{-}(\sigma)$. If each $L k^{-}(\sigma)$ is nonempty and topologically simple then the resulting $\mathbb{F}$ can have few critical simplices. The trick is to find a suitable vertex ordering function $g$. When $M$ is a compact combinatorial manifold, a good candidate is the distance function $d$.

In the Riemannian setting, the idea of creating a "Morse theory" for the distance function led to the important breakthroughs by Grove and Shiohama in [8], and Gromov in [9]. Here in the combinatorial setting we use it to show each maximum diameter $M^{3}$ is a sphere.

\subsection{Combinatorial Sphere Theorem}

Let $d_{v} \equiv d(v, \cdot)$ be our vertex-ordering function where $v$ and $w$ are vertices with $d(v, w)=$ $2 J$. In this section, the constant-set and the descending links and stars will all be with respect to $d_{v}$.

Our complete knowledge of the structure of $M^{3}$ lets us determine $d_{v}$ nearby each vertex. Using this, we prove the following lemma concerning the subcomplex $B \equiv M^{3} \backslash S t(w)$.

Lemma 6.11 For each $\sigma \in C(B)$ except $v$, a discrete Morse function $\mathbb{F}_{\sigma}$ exists on $L k^{-}(\sigma)$ whose only critical simplex is a vertex. 
$\mathbb{F}_{x}$ on $L k^{-}(x)$ for vertices $x \in C(B)$

\begin{tabular}{|c|c|c|c|c|c|c|}
\hline$\langle x, 1\rangle$ & $\langle x, 2\rangle$ & $\langle x, 3\rangle$ & $\langle x, 4\rangle$ & $\langle f, 1\rangle$ & $\langle f, 2\rangle$ & $\langle e, 1\rangle$ \\
\hline${ }^{A}$ & & $B_{1}$ & & & $\sqrt{E}$ & $\underbrace{A}_{E}$ \\
\hline
\end{tabular}

$\mathbb{F}_{\sigma}$ on $L k^{-}(\sigma)$ for higher dimensional $\sigma \in C(B)$

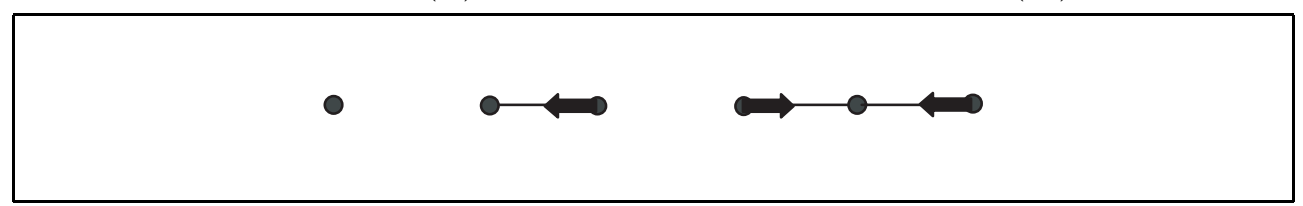

Figure 11: This figure shows the discrete Morse functions chosen on $L k^{-}(\sigma)$ for each $\sigma \in C(M)$. The simplices in $C(M)$ and their corresponding descending links $L k^{-}(\sigma)$ can be identified using Figures 7, 8 and 9. The vertex labels $A, B, C \ldots$ etc in the top table correspond to the labels in those figures.

PROof. Corollary 5.3 implies any vertex $x \in B \backslash\{v\}$ is the internal vertex of a $P_{\sigma}$. Therefore Lemma $5.4,5.5$ or 5.6 completely determines the values of $d_{v}$ on $L k(x)$. This allows us to identify all possible $\sigma \in C(B)$ by checking $d_{v}$ using the coordinates listed in Figures 7,8 or 9 . In each case $L k^{-}(\sigma)$ admits an appropriate discrete Morse function $\mathbb{F}_{\sigma}$. See Figure 11.

Now, we are ready to prove part 1 of Theorem 1.2, which we restate for your convenience.

Theorem 1.2 (Part 1) If $M$ be a positively curved combinatorial 3-manifold with vertices $v, w$ at edge distance five then $M$ is homeomorphic to a 3 -sphere.

PROOF. By Lemma 6.10 the disjoint union $\mathbb{F}=\bigsqcup_{\sigma \in C(B)} \mathbb{F}_{\sigma}^{*}$ of the induced $\mathbb{F}_{\sigma}^{*}$ forms a discrete Morse function on $B$. By Lemma 6.11 and Corollary 6.9, every simplex in $B$ except $v$ is paired by $\mathbb{F}$ into a discrete Morse arrow, making $v$ the only critical simplex of $\mathbb{F}$. Thus, since $B=M^{3} \backslash S t(w)$ is a combinatorial manifold with boundary, Theorem 6.5 implies that it is homeomorphic to a 3-ball. Since $S t(w)$ is also a 3-ball, $M^{3}$ consists of two 3 -balls glued together and is therefore a 3 -sphere as desired.

\section{Final Comments}

We mention an important corollary of Theorem 1.1. 
Corollary 7.1 Only finitely many positively curved manifolds $M^{n}$ exist for each $n$.

This immediately suggests a formidable classification problem: Which manifolds have positively curved triangulations? The answer for the $n \geqslant 4$ cases can be found in [13]. This author has learned that the $n=3$ census has recently been completed by Lutz and Sullivan in [14].

\section{References}

[1] N. Brady, J. McCammond, and J. Meier, Bounding edge degrees in triangulated 3manifolds, Proc. Amer. Math. Soc. 132 (2004), no. 1, 291-298 (electronic).

[2] Y. Cheng, Eigenvalue comparison theorems and its [sic] geometric applications, Math. Z. 143 (1975) 289-297.

[3] K. Crowley, Doctoral Thesis (Rice University), Discrete Morse Theory and the Geometry of Nonpositively Curved Simplicial Complexes. (2001).

[4] M. Elder, J. McCammond, and J. Meier, Combinatorial conditions that imply wordhyperbolicity for 3-manifolds., Topology, 42, (2003), 1241-1259.

[5] R. Forman, Morse theory for cell complexes, Adv. in Math. 134 (1998), 90-145.

[6] R. Forman, Combinatorial Differential Topology and Geometry, New perspectives in algebraic combinatorics (Berkeley, CA, 1996-97), 177-206, Math. Sci. Res. Inst. Publ., 38, Cambridge Univ. Press, Cambridge, 1999.

[7] A. Gabrielov, I. Gelfand, and M. Losik. Combinatorial computation of characteristic classes. I, II, and A local combinatorial formula for the first Pontrjagin class. Functional Anal. Appl. 9 (1975), no. 2, 103-116; ibid. 9 (1975), no. 3, 186-202 (1976); ibid. 10 (1976), no. 1, 12-15.

[8] K. Grove; K, Shiohama, A generalized sphere theorem, Ann. of Math. 106 (1977), 201-211.

[9] M. Gromov, Curvature, diameter, and Betti numbers, Comm. Math. Helvetici 56 (1981), 179-195.

[10] F. Luo and R. Stong, Combinatorics of triangulations of 3-manifolds. Trans. Amer. Math. Soc. 337 (1993), no. 2, 891-906.

[11] D.A. Stone, Sectional curvature in piecewise linear manifolds, Bull. Amer. Math. Soc., vol. 79 (1973), pp. 1060-1063.

[12] V.A. Toponogov, Riemannian spaces having their curvature bounded below by a positive number, Uspekhi Math. Nauk 14 (1959), 87-135 English Translation: Amer. Math. Soc.Transl. Ser. 37 (1964) 287-290.

[13] M. Deza, M. Dutour and M. Shtogrin, On simplicial and cubical complexes with short links, Israel Journal of Mathematics 144 (2004) 109- 124.

[14] F. H. Lutz and J. M. Sullivan: Simplicial manifolds with small valences. In preparation. 